REVISTA CIENCIAS BIOMÉ DICAS

ARTÍCULOS DE REVISIÓN

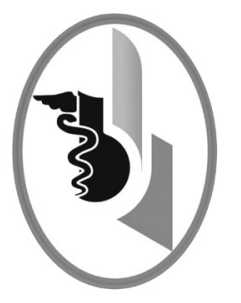

\title{
ESTRÉS OXIDATIVO, DAÑO AL ADN Y CANCER
}

\author{
OXIDATIVE STRESS, DNA DAMAGE AND CANCER
}

\author{
García-Uribe Liseth Paola ${ }^{1}$ \\ Marquéz-Lázaro Johana Patricia² \\ Viola-Rhenals Maricela ${ }^{3}$
}

Correspondencia: pao_lita2909@hotmail.com

Recibido para evaluación: mayo - 24 - 201. Aceptado para publicación: abril - 25-2015.

\section{RESUMEN}

Introducción: el cáncer es una enfermedad multifactorial, caracterizada por el crecimiento descontrolado y disperso de células anormales. Si este crecimiento y su dispersión no son controlados oportunamente, puede resultar en metástasis lo que aumenta el riesgo de muerte. El estrés oxidativo en los últimos años se ha convertido en una diana terapéutica contra esta enfermedad, debido a que se ha evidenciado su papel importante en el proceso de la carcinogénesis, de ahí que antioxidantes y proxidantes sean potenciales agentes antitumorales administrados solos o como coadyuvantes en las quimioterapias y radioterapias.

Objetivo: revisar la implicación del estrés oxidativo en los daños al ADN y en el proceso de carcinogénesis.

Metodología: revisión temática. Se realizó búsqueda informática en inglés y español en las bases de datos ScienceDirect y Scielo entre los años 2009 a 2013. Se incluyeron estudios en humanos y animales. 150 resúmenes fueron obtenidos, 50 resúmenes fueron considerados pertinentes. Todos los estudios fueron adquiridos en texto completo, revisados y citados a lo largo de la presente revisión.

Resultados: el estrés oxidativo ocurre como un desbalance entre la producción de especies reactivas y el sistema antioxidante que las neutraliza. Las especies reactivas han sido implicadas en el proceso de carcinogénesis, debido a que puede mediar la activación de vías de proliferación celular e inhibir la apoptosis, además se ha demostrado que el daño oxidativo al ADN lleva a mutaciones, favoreciendo aún más este proceso.

Conclusión: el estrés oxidativo puede ser un importante evento celular implicado en la carcinogénesis, de ahí que el uso de agentes antioxidantes y proxidantes como potenciales antitumorales constituyen una alternativa para la terapia anticancerígena, ya sean solos o como coadyuvantes. Sin embargo se requieren más estudios que permitan validar los resultados obtenidos. Rev.cienc.biomed. 2015;6(1):107-117

\section{PALABRAS CLAVE}

Cáncer; Estrés oxidativo; Antioxidantes.

1 Estudiante de Pregrado. Medicina. Grupo de Bioquímica y Biología Celular del Cáncer. Universidad de Cartagena. Colombia.

2 Químico. Magíster en Bioquímica. Grupo de Bioquímica y Biología Celular del Cáncer. Universidad de Cartagena. Colombia.

3 Químico Farmacéutico. Doctor en Ciencias Mención Bioquímica. Grupo de Bioquímica y Biología Celular del Cáncer. Universidad de Cartagena. Colombia. 


\section{SUMMARY}

Introduction: Cancer is a multifactorial disease characterized by the uncontrolled and disperse growth of abnormal cells. If this growth and its dispersion are not controlled at the appropriate time, it could result in metastasis, which increases the risk of death associated to this disease. In the last years, the oxidative stress had become a therapeutic target against this disease, due to its important role in the carcinogenesis process that has been evidenced and of there that the antioxidants and pro-oxidants are potential antitumor agents that could be administered alone or as co-adjuvants in the chemotherapies and radiotherapies.

Objective: To review the implication of oxidative stress in the DNA damages and in the carcinogenesis process.

Methods: Thematic review. An informatic search was carried out in English and Spanish, in the databases ScienceDirect and Scielo between the years 2009 and 2013. Studies in humans and animals were included. 150 summaries were obtained, 50 summaries were considered as pertinent, and of transcendence and contribution. All were acquired in full text, reviewed and cited lengthways of the present thematic review.

Results: The oxidative stress occurs as an imbalance between the production of reactive species and the antioxidant system that neutralizes them. The reactive species had been implied in the carcinogenesis process and due to they could mediate the activation of cellular proliferation ways and to inhibit the apoptosis, also it has been demonstrated that the oxidative damage to the DNA carries to mutations favoring this process.

Conclusions: The oxidative stress could be an important cellular event implied in the carcinogenesis, therefore the use of antioxidant and pro-oxidant agents as potent antitumor agents constitutes an alternative for the anticancer therapy, already be alone or as co-adjuvant. Although, more studies are required to allow validating the obtained results. Rev.cienc.biomed. 2015;6(1):107-117

\section{KEYWORDS}

Cancer; Oxidative stress; Antioxidants.

\section{INTRODUCCIÓN}

El cáncer es una enfermedad multifactorial y constituye una de las principales causas de muerte alrededor del mundo, en 2008 se le atribuyeron 7,6 millones de defunciones correspondiendo al $13 \%$ del total (1). La tasa de supervivencia varía dependiendo el tipo histológico y su localización; sin embargo, el diagnóstico precoz y la prevención juegan un rol importante para disminuir la mortalidad de esta patología $(2,3)$. En los últimos 50 años, la incidencia del cáncer ha ido en aumento, lo cual se ha atribuido principalmente al envejecimiento de la población y la difusión de agentes carcinogénicos que se encuentran en el medio ambiente (4). Dentro de los factores de riesgo asociados con el desarrollo de cáncer se han relacionado estilos de vida poco saludables, predisposición genética e infecciones por algunos virus $(1,4)$.

Los mecanismos por los cuales se desarroIla el cáncer se han estudiado durante décadas, no obstante, todavía se desconoce mucho acerca de las causas de esta patología (5). En el proceso de carcinogénesis ocurren daños a los componentes celulares como el ADN y mutaciones en diversas proteínas que controlan el ciclo celular replicando descontroladamente de las células tumorales (6).

En los últimos años se ha estudiado el rol que desempeña el estrés oxidativo en el desarrollo de la carcinogénesis, encontrándose que participa por diversos mecanismos en todas las etapas de este proceso y por lo tanto constituye un posible blanco para nuevas terapias anticancerígenas $(7,8,9)$

El objetivo de este artículo es realizar una revisión temática acerca de la implicación del estrés oxidativo en los daños al ADN y en el proceso de carcinogénesis.

\section{MATERIALES Y MÉTODOS}

Tipos de estudios: se incluyen en la revisión artículos experimentales y de revisión, estudios in vivo (humanos y ratas) e in vitro (células humanas) cuyo contenido presentaran conceptos de estrés oxidativo y cáncer. Los estudios se incluyeron sin restricción de diseño ni nivel de evidencia. 
Estrategia de búsqueda: se realizó búsqueda bibliográfica informática en ScienceDirect y Scielo. Se incluyeron estudios de todos los países, sin límite de edad ni género, en inglés y español, publicados entre 2009 y 2014.

Términos claves utilizados: se utilizaron para la revisión los términos "cáncer" and "oxidative stress".

Método de revisión: los resúmenes fueron revisados de forma separada e independiente. Se escogieron los considerados subjetivamente como pertinentes y se adquirieron las publicaciones completas. Ellas a su vez fueron revisadas y seleccionadas las que permitían cumplir con el objetivo de la revisión.

\section{RESULTADOS}

Después de la búsqueda y de eliminar las repeticiones se obtuvieron 150 resúmenes de artículos, 50 de ellos fueron considerados pertinentes, de mayor trascendencia y aporte. Todos fueron adquiridos en texto completo, revisados y citados a lo largo de la presente revisión temática.

\section{Estrés oxidativo: un desbalance media- do por producción de especies reactivas.}

El estrés oxidativo es un desbalance entre la producción de radicales libres y metabo- litos reactivos también denominados especies reactivas y su eliminación por mecanismos protectores conocidos como antioxidantes (10). Las especies reactivas de oxígeno (ERO) son metabolitos derivados del oxígeno, caracterizados por estar parcialmente reducidos, lo que le da gran avidez para aceptar electrones de otras moléculas, pudiendo afectar la estructura o función de las macromoléculas celulares, interfiriendo así con el funcionamiento celular $(11,12)$. Las ERO pueden ser endógenamente o exógenas. La principal fuente endógena es la cadena respiratoria mitocondrial, durante el metabolismo aeróbico las células producen EROs como anión superóxido $\left(\mathrm{O}_{2}^{-}\right)$, peróxido de hidrógeno $\left(\mathrm{H}_{2} \mathrm{O}_{2}\right)$ y radical hidróxilo $(\cdot \mathrm{OH})(10,13)$. Otra fuente endógena de EROs es la defensa antimicrobiana; los macrófagos y neutrófilos, los cuales liberan estas sustancias para contribuir a la eliminación de microorganismos $(11,14)$. Dentro de las fuentes exógenas de estrés oxidativo se encuentran la radiación UV, compuestos químicos (contaminantes ambientales, alcohol, cigarrillo), medicamentos y ejercicio (14). Por otro lado, bajo condiciones hipóxicas, la cadena respiratoria mitocondrial puede producir además de los EROs óxido nítrico (NO), el cual puede generar especies reactivas de nitrógeno, las cuales a su vez aumentan el estrés oxidativo y por ende los daños a la célula (10) (Figura No1).

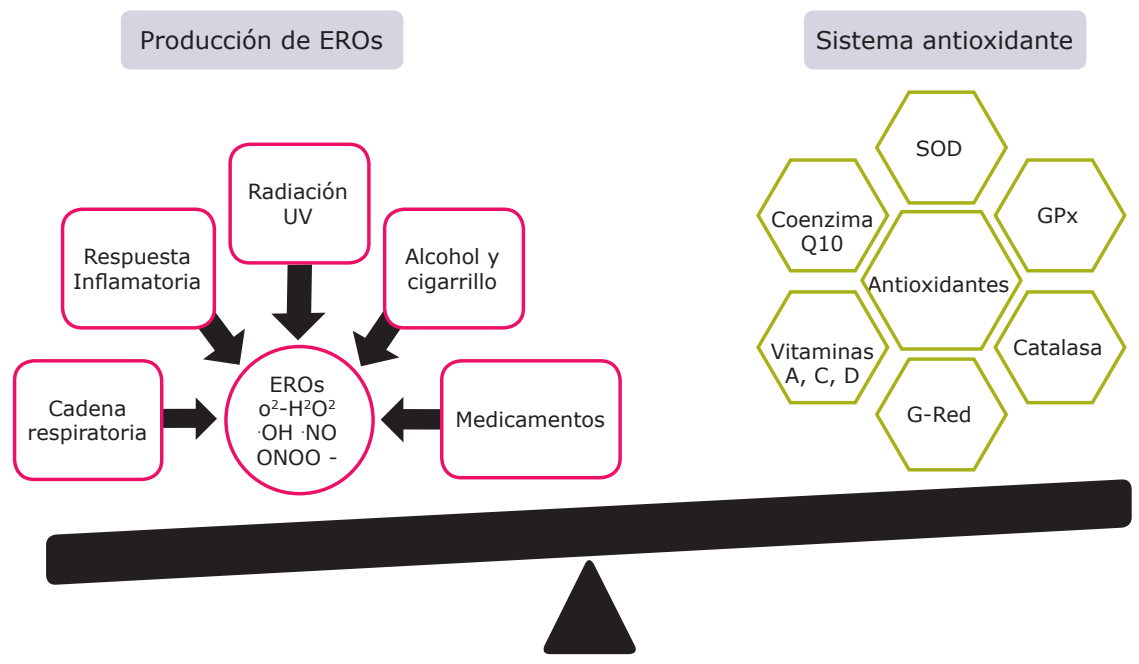

FIGURA No 1. Estrés oxidativo. Representación del balance que existe entre la producción de especies reactivas y el sistema antioxidante celular. Si este balance se pierde se genera estrés oxidativo. 
Para protegerse del ambiente pro-oxidante causado por las especies reactivas, la célula cuenta con enzimas antioxidantes, que trabajan en conjunto para llevar a cabo su destoxificación. Estas enzimas son superóxido dismutasa (SOD), glutatión peroxidasa (GPx), glutatión reductasa y catalasa (CAT). A parte del sistema endógeno antioxidante existen compuestos exógenos que cumplen esta función, actuando como antioxidantes no enzimáticos, dentro de los que se destacan glutatión (GSH), vitaminas $A, C$ y $D$, coenzima Q10, entre otros $(15,16)$.

Las EROs en condiciones de estrés pueden causar daño a los componentes celulares y llevar a la formación de productos de peroxidación lipídica, oxidación proteica y oxidación al ADN. Siendo los daños oxidativos al ADN un punto importante en esta revisión $(10,17)$.

\section{Daño oxidativo al ADN: ¿Transformación maligna?}

El daño oxidativo a esta macromolécula puede ocurrir básicamente en dos niveles: en las bases nitrogenadas o en el azúcar (desoxirribosa) (11). Cuando el ataque oxidante es a nivel de las bases púricas, las dos modificaciones más frecuentes ocurren por la adición del radical hidroxilo $(\cdot \mathrm{OH})$ a la posición C8 del anillo de la guanina, produciendo un radical que puede ser oxidado a 8 -hidroxi- ${ }^{\prime}$ -deoxiguanosina (8-OHdG) o reducido a 2,6-diamino-4-hidroxi-5-formamidopirimidina (Fapy G). Estas moléculas han mostrado ser potencialmente mutagénicas, debido a que inducen a errores durante la replicación y transversión del tipo A:T $\square$ C:G. $(12,18)$. Si la interacción del radical $\mathrm{OH}$ es con bases pirimidínicas, la modificación más común es 5,6-dihidroxi-5,6-dihidrotimina o también llamada timidín glicol (18). Figura N02. Los productos antes mencionados no resultan letales para la célula, pero son altamente mutagénicos (12).

Los daños asociados con la desoxirribosa producen una pérdida de la base nitrógenada generando así sitios AP (apurinicos o apirimidínicos) (11). Estos sitios se forman a través de la hidrólisis del enlace $\mathrm{N}$-glicosídico de los nucleótidos del ADN (mayormente es-
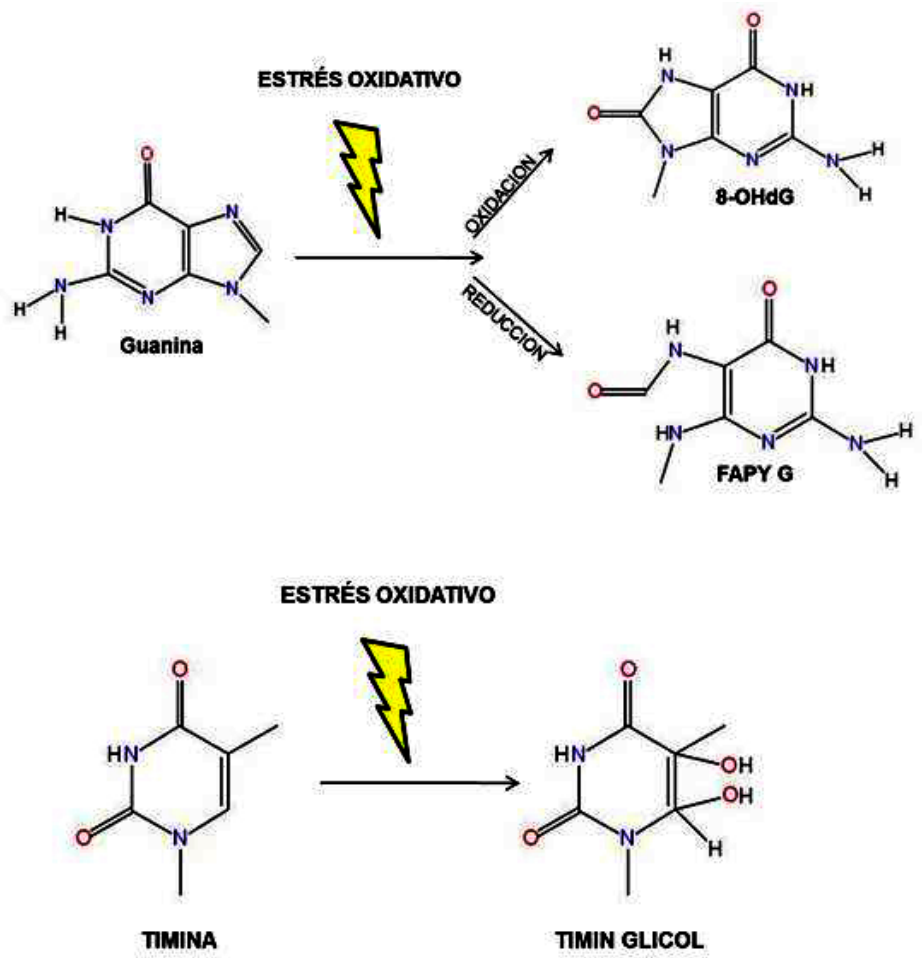

FIGURA No 2. Cambios oxidoreductivos sobre bases nitrogenadas. (A) Cambios en la guanina, en donde la adición de radical . $\mathrm{OH}$ al anillo de guanina puede generar 8-hidroxi-2' -deoxiguanosina (8-OHdG) por reacción de oxidación, o 2,6-diamino-4-hidroxi-5-formamidopirimidina (Fapy G) por una reacción de reducción. (B) Cambios en la timina por adición de radical .OH genera 5,6-dihidroxi-5,6-dihidrotimina (timidín licol) 
pontánea), de esta manera la base es removida, pero el esqueleto fosfodiester permanece intacto creando un sitio AP $(12,18)$. Característicamente los sitios a básicos que se originan debido al estrés oxidativo, surgen de la abstracción del átomo de hidrógeno por el radical $\mathrm{OH}$ en la posición $\mathrm{C} 1$ o $\mathrm{C} 4$ de la fracción 2-deoxiribosa de DNA (18).

Adicionalmente, el daño a las bases producido por estrés oxidativo y la producción de moléculas como 8-OHdG y Fapy G es considerado posible precursore de sitios AP, debido a que incrementa la labilidad hidrolítica de los enlaces $\mathrm{N}$-glucosidicos. Aunque los sitios AP no son considerados letales, cuando están presentes en altos niveles pueden interferir con las enzimas DNA polimerasa y DNA ligasa y por ende ser altamente mutagénicos $(18,19)$. La interacción de radical $\mathrm{OH}$ con el ADN puede generar en presencia de oxígeno, radicales peróxido, los cuales pueden extraer átomos de hidrógeno de las fracciones de azúcares llevando a ruptura de las hebras

de ADN (18). El resultado final in vivo de la interacción del ADN con las EROs depende de muchos factores como la reparación del ADN, los niveles de enzimas antioxidantes y la secuencia de ADN (20). Además, las EROs pueden atacar proteínas claves en la reparación del ADN y control del ciclo celular y afectar así el correcto funcionamiento de la célula $(19,20)$ (Figura No 3).

\section{Carcinogénesis: proceso favorecido por daños oxidativos al ADN}

El daño en el ADN causado por las EROs juega un papel importante en el desarrollo de la carcinogénesis; sin embargo, también está asociado con procesos de envejecimiento del organismo, por lo cual se han estudiado los efectos del estrés oxidativo sobre oncogenes, genes supresores tumorales y proliferación celular (21). La carcinogénesis es un proceso dividido principalmente en tres fases: iniciación, promoción y progresión ( $\mathrm{Fi}-$ gura No 4). Los radicales libres juegan un

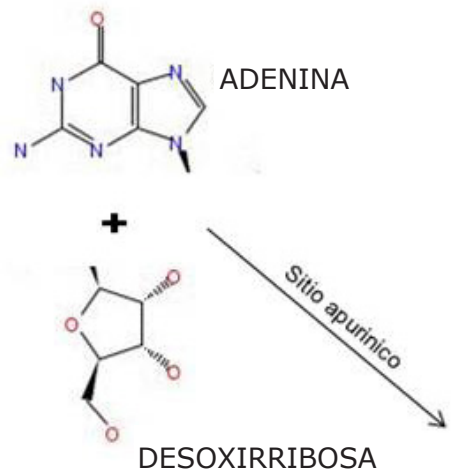

ADENOSINA

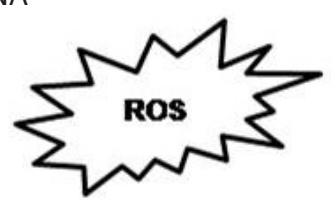

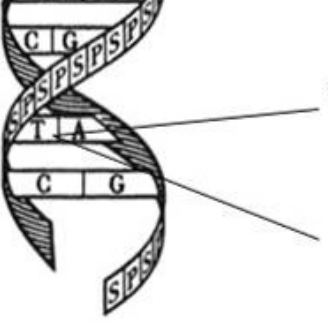

ADN

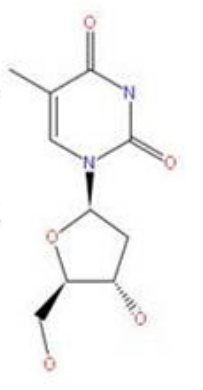

TRIMIDINA

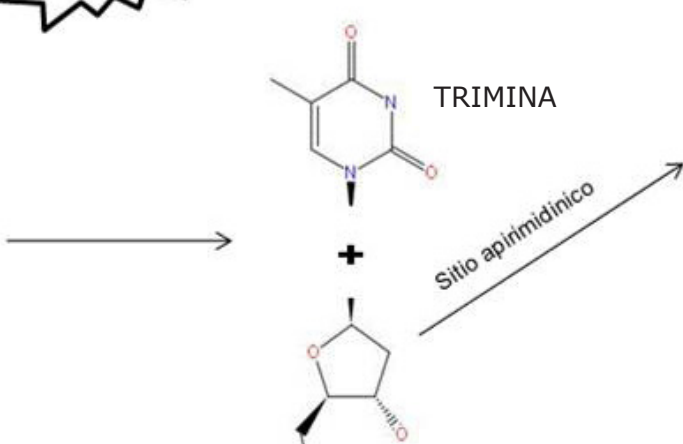

DESOXIRRIBOSA

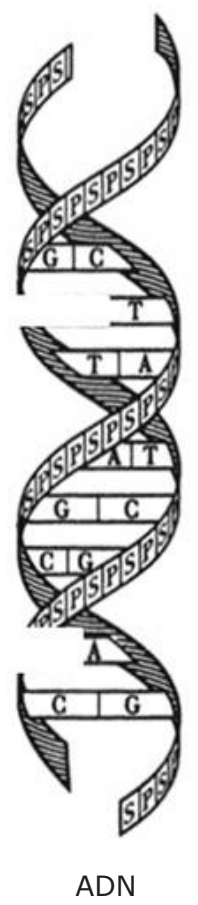

Figura No 3. Formación de sitios ap. Los sitios ap son formados como consecuencia del estrés oxidativo en la célula, en el esquema se observa los purínicos y los pirimidínicos. 
papel importante en cada fase induciendo mutaciones genéticas y activando vías de señalización que promueven la proliferación y supervivencia celular $(19,22)$.

Por un lado, durante la fase de iniciación, los radicales libres contribuyen a la transformación celular a través del daño oxidativo del ADN, anulación de los mecanismos de muerte celular programada y promoción de la expresión de oncogenes $(21,22)$. El gen supresor tumoral $p 53$ juega un papel importante en la prevención de transformación oncogénica, en condiciones normales, este gen modula la transcripción de enzimas antioxidantes como MnSOD y GPx para mantener el balance oxidativo. Cuando existe un aumento en las concentraciones de radicales libres, la proteína p53 puede desencadenar bloqueo del ciclo celular y apoptosis; sin embargo, se ha reportado que la producción excesiva de EROs puede inhibir la actividad de p53 por oxidación de los residuos de cisteína (19, 21,22).

De otro lado, los radicales libres pueden activar señales que controlan pasos fundamentales de la apoptosis, el exceso de estrés oxidativo genera productos que pueden unirse a residuos tiol o cisteína de las caspasas y al receptor CD95/Fas, lo cual lleva a inhibir esta vía de señalización. Adicionalmente, la presencia de anión superóxido $\left(\mathrm{O}_{2}^{-}\right)$aumenta intracelularmente el $\mathrm{pH}$ citosólico, lo cual desregula la activación de las caspasas e inhibe el proceso apoptótico (22).

En la fase de promoción se produce la expansión clonal de las células inicialmente mutadas a través de la inducción de la proliferación celular y la inhibición de la apoptosis, dando como resultado una lesión identificable en el tejido conocida como tumor primario (22). Los mecanismos por los cua-

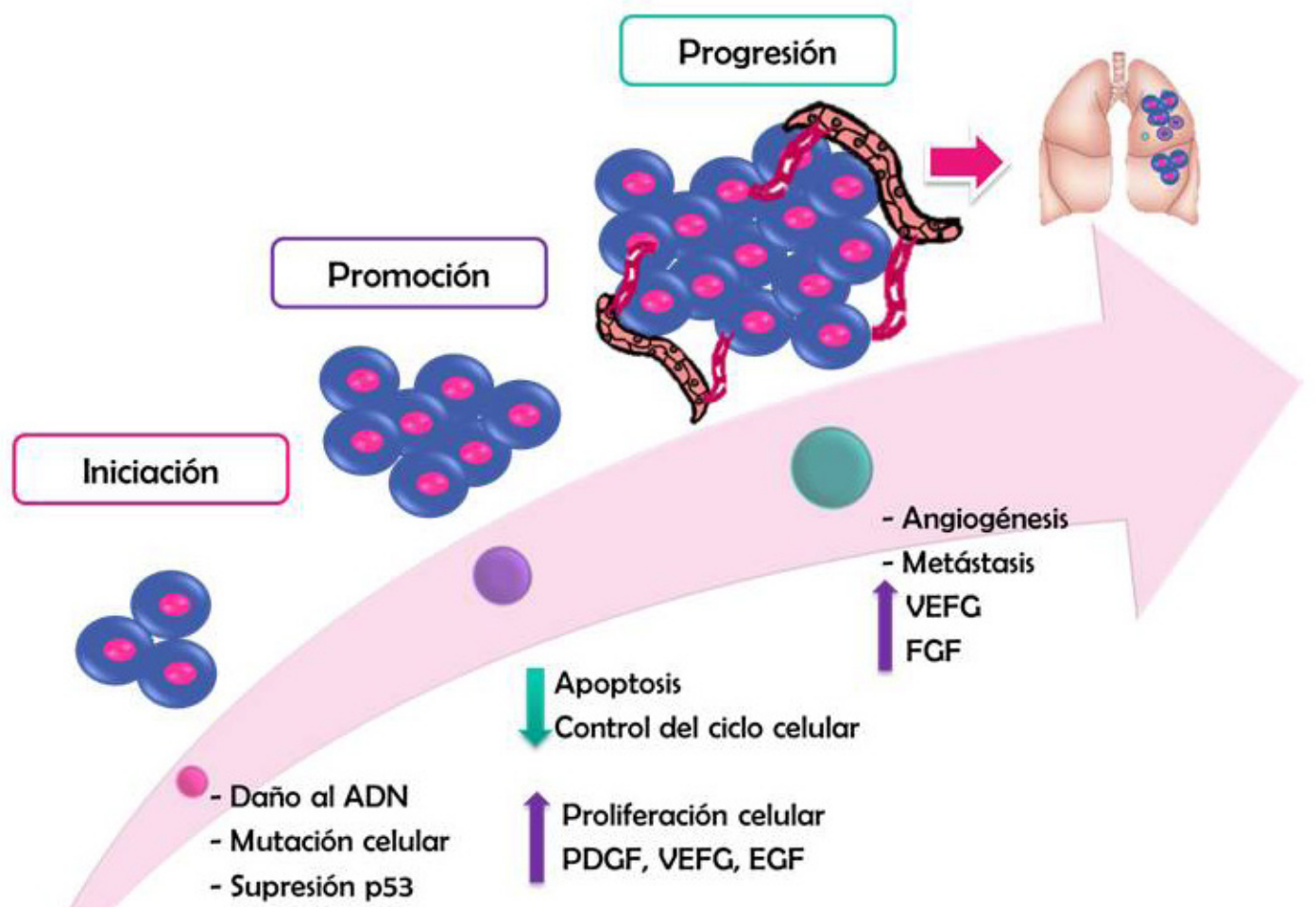

FIGURA 4. Fases de la carcinogénesis. En la Iniciación se produce el daño al ADN y por lo tanto la mutación celular, durante la promoción las células mutadas se dividen rápidamente por pérdida del control del ciclo celular y disminución de la apoptosis, finalmente en la fase de progresión las células tumorales adquieren habilidad angiogénica y metastásica con lo cual migran y colonizan tejidos distantes. 
les los radicales libres participan en este proceso incluyen interacciones con hormonas, factores de crecimiento y citoquinas para activación de diferentes vías que controlan las funciones celulares $(22,23)$. Dentro de los factores de crecimiento que se ven afectados por los radicales libres podemos mencionar el factor de crecimiento epidérmico (EGF), el factor de crecimiento derivado de plaquetas (PDGF) y el factor de crecimiento endotelial vascular (VEGF). La estimulación de alguno de los factores anteriores conlleva a un incremento transitorio en las concentraciones de EROs (22).

Las citoquinas como factor de necrosis tumoral alfa (TNF-a), interleucina $1 \beta$ (IL-1 $\beta$ ) e interferón gamma (INF-Y) pueden unirse a receptores transmembrana y transmitir señales que estimulan la producción de EROs (22). Además, el incremento de radicales libres estimula la activación de quinasas como la proteínkinasa C (PKC) la cual juega un rol muy importante en la proliferación, diferenciación, angiogénesis y apoptosis $(21,22)$.

En la fase de progresión, es la última fase de la carcinogénesis, caracterizada por cambios celulares irreversibles, las células desarrollan capacidad angiogénica, un proceso clave para el crecimiento tumoral y su aporte de oxígeno y nutrientes (22). A medida que el tumor crece en tamaño su ambiente hipóxico conlleva a la activación de las vías angiogénicas lo cual culmina en el brote de vasos sanguíneos en los tejidos circundantes al tumor. La vasculatura tumoral se caracteriza por anormalidades en las células endoteliales, pericitos y células de musculo liso, lo cual conlleva a inestabilidad y fragilidad de los vasos sanguíneos aumentando el riesgo de hemorragia (24).

Adicionalmente en esta etapa, las células tumorales adquieren la capacidad de migrar y colonizar otros tejidos en órganos distantes a través de los vasos sanguíneos, proceso conocido como metástasis (25). Para lograr una migración exitosa las células tumorales presentan una transición epitelial-mesenquimal (EMT) a través de la cual las células se desprenden de su lámina basal y reorganizan su citoesqueleto para aumentar la movilidad y migrar en el tejido circundante (22).
Existen varias vías de señalización asociadas a EROs que están implicadas en el proceso EMT, la vía del factor de crecimiento tumoral $\beta$ (TGF- $\beta$ ), ha sido de las más estudiadas. TGF- $\beta$ provoca un incremento en los niveles de EROs intracelulares por un proceso asociado con la fosforilación de Smad2. El rol de las EROs en el proceso de ETM se ve evidenciado por la regulación que ejercen en las metaloproteinasas de matriz (MMP), en especial la MMP-3, enzima que se encarga de degradar colágeno tipo II, III, IV, IX y $X$, proteoglicanos, fibronectina, laminina $y$ elastina, lo cual lleva a la degradación de matriz extracelular, jugando así un papel determinante en los procesos de angiogénesis y metástasis tumoral $(14,24)$.

\section{Blancos y fármacos basados en estrés oxidativo}

El papel del estrés oxidativo en el inicio y desarrollo de la carcinogénesis ha sido ampliamente evidenciado, lo cual ha llevado a considerar la posibilidad del uso de agentes antioxidantes y pro-oxidantes en la terapia antitumoral a fin de prevenir diferentes tipos de cáncer o ayudar en el tratamiento $(26,27)$.

Dentro de los beneficios del uso de antioxidantes se mencionan la posibilidad de reducir las dosis de los medicamentos anticancerígenos disminuyendo sus efectos adversos (26). Se ha evidenciado que la radioterapia y quimioterapia han demostrado incrementar los niveles de estrés oxidativo y afectar negativamente la calidad de vida del paciente $(28,29)$, por lo que el empleo de agentes antioxidantes pudiera ser importante en el tratamiento de éste tipo de pacientes. Con respecto a lo anterior, Tarlovsky y col (2011) encontraron que la calidad de vida de pacientes con cáncer cervicouterino mejoró en aquellas pacientes que recibían suplementos con antioxidantes (vitamina C, vitamina E, zinc y selenio) en comparación con aquellas que no lo hacían (30). Se han realizado diversos estudios buscando posibles blancos terapéuticos basados en estrés oxidativo en los tipos de cáncer más frecuentes $(31,32)$.

El uso de la vitamina C (ácido ascórbico) en la terapia anticancerígena ha sido discutido 
desde hace años y su utilidad aún es controversial (33). Estudios recientes soportan que la administración parenteral de ácido ascórbico suprime el crecimiento de ciertas líneas tumorales ya que se alcanzan las concentraciones plasmáticas necesarias para que este cumpla su actividad citolítica $(34,35)$.

Verrax y Calderón (2009) evaluaron la actividad antitumoral del ácido ascórbico in vitro usando líneas tumorales como T24 (vejiga), HepG2 (hígado) y MCF7 (seno), que fueron incubadas en presencia de concentraciones de vitamina $\mathrm{C}$ similares a las obtenidas en plasma después de su administración parenteral, encontrándose que se disminuía considerablemente la tasa de crecimiento tumoral. Estos resultados soportan la idea que el ácido ascórbico induce la producción de peróxido de hidrógeno extracelular llevado a muerte por necrosis de la célula cancerígena (34).

La radioterapia constituye la piedra angular en el tratamiento del cáncer de próstata, su característica principal se basa en el daño oxidativo a las células tumorales por generación de EROs. Sin embargo, ciertas líneas celulares, principalmente las metastásicas han demostrado ser resistentes a la citotoxicidad de la radioterapia, sugiriendo que el sistema antioxidante en estas células podría jugar un papel importante en su resistencia (36).

Freitas y col (2012) realizaron un ensayo clínico para valorar la inhibición del crecimiento celular inducida por peróxido de hidrógeno $\left(\mathrm{H}_{2} \mathrm{O}_{2}\right)$ en tres líneas celulares, HPV10 y PC3 (de cáncer de próstata localizado y metastásico) y RWPE1 (células de epitelio prostático sano). En este estudio se encontró que el crecimiento celular de la línea PC3 no era inhibida por $\mathrm{H}_{2} \mathrm{O}_{2}$, mientras que HPV10 y RWPE1 mostraban muerte celular por necrosis e inhibición de la tasa de crecimiento. Adicionalmente, las células PC3 mostraron niveles mayores de glutatión (GSH) y actividad de glutatión reductasa en comparación con las otras dos líneas celulares, por lo cual fueron tratadas con Dietilmaleato (DEM), encontrándose que después de 24 horas presentaban disminución de los niveles de GSH y de actividad de glutatión reductasa, concomitantemente se observó una reducción de la proliferación celular y aumento de la citotoxicidad mediada por $\mathrm{H}_{2} \mathrm{O}_{2}$ (36). Estos resultados sugieren la posibilidad de utilizar agentes que modifiquen la activad de glutatión reductasa en el tratamiento de cáncer de próstata resistente a radioterapia.

El papel del cobre en la carcinogénesis ha sido ampliamente estudiado, se ha reportado que in vitro el cobre estimula la movilidad de las células endoteliales e induce la síntesis de fibronectina, una glicoproteína asociada con la angiogénesis (37). La utilización de cobre reducido como terapia anticancerígena ha sido un amplio campo de investigación, el uso de quelantes de cobre como D-penicilamina (D-pen) ha demostrado inhibir la angiogénesis in vitro e in vivo (38). La incubación de células endoteliales con D-pen en presencia de cobre, ha mostrado producir muerte celular, debido a la generación de EROs, principalmente por $\mathrm{H}_{2} \mathrm{O}_{2}$, lo cual soporta la capacidad antiangiogénica de los quelantes de cobre y su posible utilidad como terapia anticancerígena (38).

El factor nuclear $\mathrm{kB}$ (NF-kB) juega un rol importante en muchas vías de señalización, incluidas las que activan los procesos inflamatorios y carcinogénesis. Recientemente se ha encontrado que la vía NF-kB es crítica para mantener los niveles de GSH y que su activación constitutiva en diversos tumores puede estar implicada en la resistencia a la quimioterapia (39). La activación constitutiva de NF-KB está presente en el $15-20 \%$ de todos los cánceres y a estas células cancerígenas se les llama NF-kB positivas. Meng y col (2010) trataron células de cáncer de próstata NF-KB positivas con JSH-23, un inhibidor de NF-KB, encontrando que se reducían los niveles de GSH en estas células y se mejoraba la respuesta al tratamiento con arsénico, un antitumoral que actúa por producción de EROs (39).

La bleomicina es un agente quimioterapéutico utilizado comúnmente en el tratamiento de linfomas y tumor testicular de células germinales, sin embargo su uso terapéutico ha sido limitado debido a su toxicidad 
pulmonar causada por producción de EROs (40). Corty col (2012) evaluaron el uso de $\mathrm{N}$-acetilcisteína en conjunto con bleomicina en células de cáncer testicular, evidenciando que los niveles de marcadores de estrés oxidativo y peroxidación lipídica disminuían. Estos resultados sugieren que el uso de $\mathrm{N}$ acetilcisteína podrían disminuir los efectos adversos de la bleomicina producidos por incremento del estrés oxidativo a nivel pulmonar (40).

Recientemente se ha estudiado el papel del hierro en la progresión del cáncer. Estos estudios sugieren que los bajos niveles de ferritina y la presencia de anemia contribuyen a la angiogénesis tumoral por medio de regulación del factor inducible por hipoxia (HIF). Lo anterior propone que el hierro podría ser usado como terapia antitumoral. Se requieren más estudios al respecto $(41,42)$.

Ciertos compuestos naturales han sido estudiados como coadyuvantes en el tratamiento anticancerígeno. Kedzierskay col (2012) estudiaron el efecto del extracto de Aronia melanocarpa (Anorox) en pacientes con cáncer de seno observando que los niveles de estrés oxidativo disminuían en estas pacientes después de la cirugía o varias fases de quimioterapia $(44,45)$.

Otro compuesto natural estudiado como antitumoral es el extracto de semilla de uva. Raina y col (2013) estudiaron este compuesto en dos líneas celulares de cáncer de vejiga T24 y HTB9 encontrando que se producía inhibición de la viabilidad celular mediada por apoptosis (46).

Los fármacos usados para quimioterapia tienen múltiples vías de acción, recientemente se ha investigado si algunos de sus efectos anticancerígenos son mediados por estrés oxidativo. Ciplastino (CIS) y Zidovudina (AZT) son dos agentes quimioterapéuticos usados frecuentemente en terapia antitumoral, y sus efectos son producidos por disfunción mitocondrial. Estudios recientes reportan que estos efectos son producidos por estrés oxidativo aunque es necesario realizar más investigación al respecto (47).
Debido a que la célula cancerígena muestra aumento del metabolismo de glucosa, se ha considerado el uso de inhibidores de glucosa para potenciar el efecto de algunos compuestos quimioterapéuticos como el $\mathrm{Pa}-$ clitaxel (PTX). Se ha reportado que la combinación de inhibidores de glucosa más PTX fue más tóxica para las células tumorales en cáncer de mama, sin embargo no lo fue para las células mesenquimales, ni epiteliales sanas (48). Adicionalmente, se encontró que la combinación de estos agentes producía incremento en los niveles de $\mathrm{H}_{2} \mathrm{O}_{2}$, lo cual sugiere que la vía por la cual se produce la muerte celular está mediada por estrés oxidativo (48).

Se han realizado investigaciones acerca del rol que juegan el ejercicio y los estilos de vida saludable en la prevención y tratamiento del cáncer (49). Estudios in vitro reportan que una nutrición adecuada acompañada de actividad física induce regulación de varios metabolitos séricos como factor de crecimiento insulínico tipo I (IGF-1), llevando a reducción de la proliferación celular y aumento de la apoptosis en células tumorales de cáncer de próstata $(50,51)$.

\section{CONCLUSIÓN}

El rol del estrés oxidativo y el daño al ADN en la carcinogénesis han sido ampliamente estudiados, lo cual ha expandido las opciones de blancos terapéuticos contra esta patología. Se han realizado muchos estudios con el fin de encontrar nuevas terapias efectivas basadas en estrés oxidativo, sin embargo es necesario realizar más estudios con el fin de demostrar la efectividad de las mismas, pudiendo deberse a que la célula cancerígena es estructuralmente y genéticamente idéntica a una célula normal lo que dificulta encontrar tratamientos específicos y selectivos. Todo esto sugiere que es necesario entender el proceso oxidativo no desde el daño genético sino sobre el daño bioquímico.

CONFLICTO DE INTERESES: ninguno que declarar.

FINANCIACION: recursos propios de los autores. 


\section{REFERENCIAS BIBLIOGRÁFICAS}

1. Organización Mundial de Salud. Centro de prensa. Cáncer [online]. Febrero 2013 [citado Julio 26 2013]. Disponible en: http//www.who.int/mediacenter/factsheets/fs297/es.

2. Deppen S, Aldrich M, Hartge P, Berg C, Colditz G, Petitti D, Hiatt R. Cancer screening: The Journey from Epidemiology to Policy. Ann Epidemiol. 2012;22(6):439-445.

3. Bassily MN, Wilson R, Pompei F, Burmistrov D. Cancer survival as a function of age at diagnosis: A study of the Surveillance, Epidemiology and End Results database. Cancer Epidemiol.2010;34(6):667-81.

4. Ziech D, Franco R, Pappa A, MalamouV, Georgakila S, Georgakilas A et al. The role of epigenetics in environmental and occupational carcinogenesis. Chem Biol Interact. 2010;188(2):340-349.

5. Kutanzi K, Koturbash I, Bronson R, Pogribny I, Kovalchuck O. Imbalance between apoptosis and cell proliferation during early stages of mammary gland carcionogenesis in ACI rats. Mutat Res. 2010;694(1-2):1-6.

6. Beckman R. Efficiency of carcinogenesis: Is a mutator phenotype inevitable? Semin Cancer Biol. 2010;20(5):340-52.

7. Ziech D, Franco R, Pappa A, Panayiotidis M. Reactive Oxygen Species (ROS) - Induced genetic and epigenetic alterations in human carcinogenesis. Mutat Res. 2011;711(1-2):16773.

8. Rodríguez A, Llacuachaqui M, Pardo G, Royer R, Larson G, Weitzel J et al. BRCA1 and BRCA2 mutations among ovarian cancer patients from Colombia. Gynecol Oncol. 2012;124(2):236-43.

9. Lee J, Son Y, Pratheeshkumar P, Shi X. Oxidative stress and metal carcinogenesis. Free Radic Biol Med. 2012;53(4):742-57.

10. Reuter S, Gupta S, Chaturvedi M, Aggarwal B. Oxidative stress, inflammation, and cancer: How are they linked?. Free Radic. Biol. Med. 2010;49(11):1603-16.

11. Garcia B, Saldaña A, Saldaña L. El estrés oxidativo y los antioxidantes en la prevención del cáncer. Rev. Hab. Cienc. Med. 2013;12(2):187-196.

12. Ziech D, Franco R, Pappa A, Malamou-Mitsi V, Georgakila S, Georgakilas A, Panayiotidis, Schoneveld $\mathrm{O}$. The role of reactive oxygen species and oxidative stress in environmental carcinogenesis and biomarker development.Chem.-Biol. Interact. 2010;188(2):334-9.

13. Battisti V, Maders L, Bagatini M, Reetz L, Chiesa J, Battisti I, et al. Oxidative stress and antioxidant status in prostate cancer patients: Relation to Gleason score, treatment and bone metastasis. Biomed.Pharmacother. 2011;65(7):516-24.

14. Sosa V, Moliné T, Somoza R, Paciucci R, Kondoh H, Lleonart M. Oxidative stress and cancer: A overview. Ageing Res. Rev. 2013;12(1):376-90.

15. Dong C, Liang HU, Ling Z, Shuang Z, QuinHui M, QuinBai G, et al. Association of Catalase Genotype with Oxidative Stress in the Predication of Colorectal Cancer: Modification by Epidemiological Factors. Biomed Environ Sci. 2012;25(2):156-62.

16. Agalliu I, Kirsh V, Kreiger N, Soskolne C, Rohan T. Oxidative balance score and risk of prostate cancer: Results from a case-cohort study. Cancer Epidemol. 2011;35(4):353-61.

17. Marsova Z, Eken A, Matevska N, Erdem O, Sayal A, Savaser A, et al. Increased oxidative/ nitrosative stress and decreased antioxidant enzyme activities in prostate cancer. Clin. Biochem. 2009;42(12):1228-35.

18. Krystona T, Georgieva A, Pissis, Georgakilasa A. Role of oxidative stress and DNA damage in human carcinogenesis. Mutat. Res. 2011;711(1-2):193-201.

19. Vera L, Ramírez M, Pérez P, Granados S, Battino M, Quiles J, et al. Long-term effects of systemic cancer treatment on DNA oxidative damage: The potential for targeted therapies. Cancer Lett. 2012;327(1-2): 134-41.

20. Khandrika L, Kumar B, Koul S, Maroni P, Koul H. Oxidative stress in prostate cancer. Cancer Lett. 2009;282(1):125-136.

21. Vera L, Sánchez P, Ramírez C, Ramírez C, Granados S, Lorente J, Quiles J. Free radicals in breast carcinogenesis, breast cancer progression and cancer stem cells. Biological bases to develop oxidative-based therapies. Crit. Rev. Oncol Hematol. 2011;80(3):347-68.

22. Mena S, Ortega A, Estrela JM. Oxidative stress in environmental-induced carcinogenesis. Mutat. Res. 2009;674(1-2):36-44.

23. Georgakila A. Oxidative stress, DNA damage and repair in carcinogenesis: Have we established a connec tion? Cancer Lett.2012;327(1-2):3-4.

24. Hoff $\mathrm{P}$, Machado K. Role of angiogenesis in the pathogenesis of cancer. Cancer Treat Rev. 2012;38(7):825-33.

25. Matsudo M, Shimomura I. Increased oxidative stress in obesity: Implications for metabolic syndrome, diabetes, hypertension, dyslipidemia, atherosclerosis, and cancer. Obes Res Clin Pract. 2013; 7(5):e330-e341.

26. Rohenkohl C, Carniel A, Colpo E. Antioxidants consumption during chemotherapy treatment. Arq Bras Cir Dig. 2011;24:107-112.

27. Mourao de Farias J, Siqueira F, Botelho S, Ribeiro A, Leitao P. Oxidative stress parameters in women with breast cancer undergoing neoadjuvant chemotherapy and treated with nutraceutical doses of oral glutamine. Acta Cir.Bras. 2011;26:82-87. 
28. Seibold P, Hall P, Schoof N, Nevannlina H, Heikkinen T, Benner A, et al. Polymorphisms in oxidative stress-related genes and mortality in breast cancer patients e Potential differential effects by radiotherapy? Breast. 2013; 22(5):817-23.

29. Skiöld S, Naslund I, Brehwens K, Anderson A, Wersall P, Lidbrink E. Radiation-induced stress response in peripheral blood of breast cancer patients differs between patients with severe acute skin reactions and patients with no side effects to radiotherapy. Mutat. Res. 2013;756(1-2):152-7.

30. Fuchs V, Bejarano M, Gutiérrez G, Casillas A, López J, Ceballos G. Efecto de la suplementación con antioxidantes sobre el estrés oxidativo y la calidad de vida durante el tratamiento oncológico en pacientes con cáncer cérvico uterino. Nutr Hosp. 2011;26:819-26.

31. Vieira F, Di Prieto P, Boaventura B, Ambrosi C, Rockenbach G, et al. Factors associated with oxidative stress in women with breast cancer. Nutr Hosp. 2011;26:528-536.

32. Rockenbach G, Vieira F, Di Prieto P, Boaventura B, Ambrosi C, Crippa G, et al. Dietary intake and oxidative stress in breast cancer: before and after treatments. Nutr Hosp. 2011;26:737744.

33. Kedzierzka M, Olas B, Wachowics B, Jeziorski A, Piekarsky J. Relationship between thiol, tyrosine nitration and carbonyl formation as biomarkers of oxidative stress and changes of hemostatic function of plasma from breast cancer patients before surgery. Clin Biochem. 2012;45:231-236

34. Verrax J, Calderon P. Pharmacologic concentrations of ascorbate are achieved by parenteral administration and exhibit antitumoral effects Free Radic. Biol Med. 2009;47:32-40.

35. Chen P, Stone J, Sullivan G, Drisko J, Chen Q. Anti-cancer effect of pharmacologic ascorbate and its interaction with supplementary parenteral glutathione in preclinical cancer models. Free Radic. Biol. Med. 2011;51:681-7.

36. Freitas M, Baldeiras I, Proeca T, Alves V, Mota-Pinto A, Sarmento-Ribeiro A. Oxidative stress adaptation in aggressive prostate cancer may be counteracted by the reduction of glutathione reductase. FEBS Open Bio 2. 2012;2:119-28.

37. Hindo S, Frezza M, Tomco D, Heeg MJ, Hryhorczuk L, McGarvey B, et al. Metals in anticancer therapy: Copper(II) complexes as inhibitors of the $20 \mathrm{~S}$ proteasome. Eur. J. Med. Chem. 2009;44:4353-61.

38. Gupte A, Mumper R. Elevated copper and oxidative stress in cancer cells as a target for cancer treatment. Cancer Treat. Rev. 2009;35:32-46.

39. Meng Q, Peng Z, Chen L, Si J, Dong Z, Xia Y. Nuclear Factor-kB modulates cellular glutathione and prevents oxidative stress in cancer cells. Cancer Lett. 2010;299:45-53.

40. Cort A, Ozdemir E, Timur M, Ozben T. Effects of N-acetyl-L-cysteine on bleomycin induced oxidative stress in malignant testicular germ cell tumors. Biochimie. 2012;94:2734-39.

41. Foy S, Lahabsetwar V. On the irony: Iron as a cancer cause or cure? Biomaterials. $2011 ; 32: 9155-8$

42. Jian J, Yang Q, Dai J, Eckard J, Axelrod J, Smith J, et al. Effects of iron deficiency and iron overload on angiogenesis and oxidative stress-a potential dual role for iron in breast cancer. Free Radical Biology \& Medicine. 2011;50:841-7.

43. Ziech D, Anestopoulos I, Hanafi R, Voulgaridou G, Franco R, Georgakilas A. Pleiotrophic effects of natural products in ROS induced carcinogenesis: The role of plant-derived natural products in oral cancer chemoprevention. Cancer Lett. 2012;327:16-25.

44. Kedzierska M, Olas B, Wachowicz B, Glowacki R, Bald E, Czernek U. Effects of the commercial extract of aronia on oxidative stress in blood platelets isolated from breast cancer patients after the surgery and various phases of the chemotherapy. Fitoterapia.2012;86:310-317.

45. Sharif T, Stambouli M, Burrus B, Emhemmed F, Dandache I, Auger C. The polyphenolic-rich Aroniamelanocarpa juice kills teratocarcinomal cancer stem-like cells, but not their differentiated counterparts. J. Funct. Food..2013;5:1244-52.

46. Raina K, Tyagi A, Kumar D, Agarwal R, Agarwal C. Role of oxidative stress in cytotoxicity of grape seed extract in human bladder cancer cells. Food Chem. Toxico.2013; 61:187-195.

47. Mattson D, Ahmad I, Dayal D, Parsons A, Aykin-Burns N, Li L, et al. Cisplatin combined with zidovudine enhances cytotoxicity and oxidative stress in human head and neck cancer cells via a thiol-dependent mechanism. Free Radic. Biol. Med. 2009;46:232-37.

48. Hadzic T, Aykin-Burns N, Zhu Y, Coleman M, Leick K, Jacobson G, Spitz D. Paclitaxel combined with inhibitors of glucose and hydroperoxide metabolism enhances breast cancer cell killing via H2O2-mediated oxidative stress. Free Radic. Biol. Med. 2010;48:1024-1033.

49. Jones L, Eves N, Spasojevic I, Wang F, II'yasova D. Effects of Aerobic Training on Oxidative Status in Postsurgical Non-Small Cell Lung Cancer Patients: A Pilot Study. Lung Cancer. $2011 ; 72: 45-51$

50. Rebillard A, Lefeuvre-Orfila L, Gueritat J, Cillard J. Prostate cancer and physical activity: Adaptive response to oxidative stress. Free Radic. Biol. Med. 2013;60:115-24.

51. Fang J, Seki T, Maeda H. Therapeutic strategies by modulating oxygen stress in cancer and inflammation. Adv Drug Deliv Rev. 2009;61:290-302. 